\title{
Use of bicistronic vectors in combination with flow cytometry to screen for effective small interfering RNA target sequences.
}

\section{$\operatorname{AUTHOR}(S)$ :}

Kamio, Naoka; Hirai, Hideyo; Ashihara, Eishi; Tenen, Daniel G; Maekawa, Taira; Imanishi, Jiro

\section{CITATION:}

Kamio, Naoka ... [et al]. Use of bicistronic vectors in combination with flow cytometry to screen for effective small interfering RNA target sequences.. Biochemical and biophysical research communications 2010, 393(3): 498-503

\section{ISSUE DATE:}

2010-03-12

URL:

http://hdl.handle.net/2433/126634

\section{RIGHT:}

(C) 2010 Elsevier B.V.; This is not the published version. Please cite only the published version.; この論文は出版社版でありません。引用の際に は出版社版をご確認ご利用ください。 
Methods Online

\section{Use of bicistronic vectors in combination with flow cytometry to screen for effective small interfering RNA target sequences}

Naoka Kamio ${ }^{1,2}$, Hideyo Hirai ${ }^{1,2, *}$, Eishi Ashihara ${ }^{1}$, Daniel G. Tenen ${ }^{3,4}$, Taira Maekawa ${ }^{1}$ and Jiro Imanishi $^{2}$

${ }^{1}$ Department of Transfusion Medicine and Cell Therapy, Kyoto University Hospital, 54 Kawahara-cho, Shogoin, Sakyo-ku, Kyoto 606-8507, Japan

${ }^{2}$ Department of Microbiology and Immunology, Kyoto Prefectural University of Medicine, 465 Kajii-cho, Kamigyo-ku, Kyoto 606-8566, Japan

${ }^{3}$ Harvard Stem Cell Institute, Harvard Medical School, Boston, MA02115

${ }^{4}$ Cancer Science Institute, National University of Singapore, Singapore 117456

${ }^{*}$ Corresponding author

Department of Transfusion Medicine and Cell Therapy, Kyoto University Hospital, 54 Kawahara-cho, Shogoin, Sakyo-ku, Kyoto 606-8507, Japan

Tel.: +81-75-751-3630

Fax: +81-75-751-4283

e-mail: hhirai@kuhp.kyoto-u.ac.jp

Keywords: bicistronic vector, flow cytometry, siRNA, green fluorescent protein 


\section{Abstract}

The efficacy and specificity of small interfering RNAs (siRNAs) are largely dependent on the siRNA sequence. Since only empirical strategies are currently available for predicting these parameters, simple and accurate methods for evaluating siRNAs are needed. To simplify such experiments, target genes are often tagged with reporters for easier readout. Here, we used a bicistronic vector expressing a target gene and green fluorescent protein (GFP) to create a system in which the effect of an siRNA sequence was reflected in the GFP expression level. Cells were transduced with the bicistronic vector, expression vectors for siRNA and red fluorescent protein (RFP). Flow cytometric analysis of the transduced cells revealed that siRNAs for the target gene silenced GFP from the bicistronic vector, but did not silence GFP transcribed without the target gene sequence. In addition, the mean fluorescence intensities of GFP on RFP-expressing cells correlated well with the target gene mRNA and protein levels. These results suggest that this flow cytometry-based method enables us to quantitatively evaluate the efficacy and specificity of siRNAs. Because of its simplicity and effectiveness, this method will facilitate the screening of effective siRNA target sequences, even in high-throughput applications. 


\section{Introduction}

RNA interference is a sequence-specific posttranscriptional gene-silencing process induced by short double-stranded RNA $(1,2)$. This evolutionally conserved phenomenon is widely applied to the functional analysis of genes and to therapeutic trials (3-7). The specificity and efficacy of gene silencing with short interfering RNAs (siRNA) are largely dependent on the siRNA sequence. Accumulating evidence suggests that only a limited number of siRNAs are capable of inducing highly effective target gene silencing in a sequence-specific manner (8-11). Many algorithms and guidelines have been developed for the design of effective siRNA sequences (12-16). However, predicting siRNA efficacy and specificity remains empirical and requires experimental verification. Therefore, simple and accurate methods for evaluating siRNAs are required.

To directly measure the protein or mRNA levels of a target gene, gene-specific reagents, such as primers or antibodies, and precisely optimized experimental conditions for each target gene are necessary. To avoid such complicating steps, target genes can instead be tagged with various reporter genes for easier readout. When a target gene sequence is followed by the combined sequences of an internal ribosome entry site (IRES) and green fluorescence protein (GFP) in an expression vector, then the target gene and GFP are transcribed as a single mRNA and are translated independently in the cell $(17,18)$. Once an siRNA against the target gene is transduced together with the bicistronic expression vector, the siRNA will essentially destroy the entire mRNA, including the target gene and GFP. The GFP expression level is then expected to reflect the efficacy of the siRNA (Figure 1).

Here, we utilized a GFP-expressing bicistronic vector to report the effect of an siRNA, and investigated whether the siRNA efficiency and specificity could be quantitatively monitored by flow cytometry. 


\section{Materials and Methods}

Cell culture

HEK293 cells were grown in Dulbecco's minimum essential medium supplemented with $10 \%$ fetal calf serum (FCS) at $37^{\circ} \mathrm{C}$ in $100 \%$ humidified air.

\section{si RNA design}

siRNA sequences against the mouse cyclic AMP-responsive element modulator (CREM) gene were designed using the online siRNA design software programs siRNA Target Finder (Ambion; http://www.ambion.com/jp/techlib/misc/siRNA_finder.html) and siDESIGN Center (Thermo Scientific; http://www.dharmacon.com/DesignCenter/DesignCenterPage.aspx). We chose three different 19-bp sequences that follow AA and contain GC sequences at less than a 50\% frequency. The sequences were as follows: CREM\#1, ggcaaatgaccatggaaa; CREM\#2, gtaattgattcgcataaac; and CREM\#3, gaagcaactcgcaagcggg. The siRNA sequences against firefly luciferase (GL3) and GFP were cttacgctgagtacttcga and caagctgaccctgaagttc, respectively.

\section{Vector construction}

Total RNA was extracted from a C57BL/6 mouse testis and reverse transcribed using the ReverTraAce kit (TOYOBO, Japan). The mouse CREM gene was amplified by PCR from the cDNA using the following primers: sense, gcgaattcatgagcaaatgtggcaggaaaaagtatatgagg; and anti-sense, ggctcggttactctgctttatggcaataa. The PCR product was digested with EcoRI and XhoI and cloned into the EcoRI-XhoI site of the retrovirus vector MSCV-IRES-GFP (pMIG). For red fluorescence protein (RFP) expression, pDsRed-Monomer-Hyg-N1 (pRFP, Clontech, CA) was used. The SiVM2 vector, in which a short hairpin RNA is driven by a human H1 promoter, was used for siRNA expression (19). 
We used the following oligonucleotides encoding mouse CREM-specific siRNAs: (CREM\#1), 5'-TCC CGG CAA ATG ACC ATG GAA ACT TCA AGA GAG TTT CCA TGG TCA TTT CCC T-3' and 5'-GGC AAA TGA CTG GAA ACT CTC TTG AAG TTT CCA TGG TCA TTT GCC-3'; (CREM\#2), 5'-TCC CGT AAT TGA TTC GCA TAA ACT TCA AGA GAG TTT ATG CGA ATC AAT TAC T-3' and 5'-GTA ATT GAT TCG CAT AAA CTC TCT TGA AGT TTA TGC GAA TCA ATT AC-3'; and (CREM\#3), 5'-TCC CGA AGC AAC TCG CAA GCG GGT TCA AGA GAC CCG CTT GCG AGT TGC TTC T-3' and 5'-GAA GCA ACT CGC AAG CGG GTC TCT TGA ACC CGC TGC GAT TGC TTC-3'. These oligonucleotides were annealed and subcloned downstream of the H1 promoter using BbsI and XcmI in the SiVM2 vector (19).

Transfection

HEK293 cells were transduced with various combinations of plasmid vectors using the Lipofectamine LTX and Plus reagents (Invitrogen, CA) according to the manufacturer's protocol. Briefly, cells were seeded 24-h prior to transfection into a 6-well plate at a density of $1 \times 105 /$ well. One microgram of DNA was mixed with $2.5 \mu$ of Lipofectamine LTX and $1 \mu 1$ of Plus reagent in $200 \mu \mathrm{l}$ of OPTI-MEM medium, incubated at room temperature for $30 \mathrm{~min}$, and then added to each well of the 6-well plate. The fluorescence of GFP and RFP were monitored using an Olympus IX71 microscope, and data were captured with DP controller/Manager software (Olympus, Japan).

Flow cytometry

Twenty-four hours after transfection, the cells were washed with phosphate buffered saline (PBS), treated with $0.05 \%$ trypsin/EDTA, and resuspended in PBS supplemented with $2 \%$ FCS. Flow cytometric analysis was carried out using FACSCalibur (BD). To calibrate our flow cytometry results, mock-transfected cells and cells transduced with either pMIG or pRFP alone were included 
with every experiment. Data were analyzed using FlowJo software (Tree Star Inc.).

Real-time PCR

RNA was extracted from cells using the RNeasy Micro kit (Qiagen) according to the manufacturer's protocol, and was reverse transcribed using a random hexamer primer. Quantitative PCR was performed using the Light Cycler Taqman Master kit (Roche). The CREM expression was normalized to GAPDH expression. The primers and probes used were as follows: (mouse CREM) left, gctgaggctgatgaaaaaca, right, gccacacgattttcaagaca, universal probe library (UPL) \#4; (GAPDH) left, tgtccgtcgtggatctac, right, cctgcttcaccaccttcttg, UPL \#80.

\section{Western blotting}

We used anti-CREM (sc-440 X, SantaCruz), anti-actin (sc7210, SantaCruz), and HRP-conjugated anti-rabbit (sc-2317, SantaCruz) antibodies for Western blotting. Band intensities were quantified using Image J software (http://rsb.info.nih.gov/ij/). 


\section{Results}

To verify that the GFP expression level could be evaluated by flow cytometry, we first examined siRNA directly targeted to GFP. An MSCV-based vector (MSCV-IRES-GFP: pMIG) was utilized as our GFP expression vector. An siRNA sequence against GFP was cloned into the siVM2 expression vector, in which siRNA was driven by a human H1 promoter (siVM2GFP) (19). A red RFP monomer expression vector (pDsRed-Momoner-Hyg-N1: pRFP) was used to normalize the transduction efficiency.

HEK293 cells were transduced with pRFP and pMIG with or without siVM2GFP, and the GFP and RFP expression levels were evaluated by fluorescent microscopy and flow cytometry $24 \mathrm{~h}$ after transfection (Fig. 2A and B, respectively). When the RFP expression vector was co-transduced with GFP, most RFP-positive cells expressed GFP, as expected (middle panels in Fig. 2A and B). The GFP expression was highly suppressed when siVM2GFP was co-transduced (bottom panels in Fig. 2A and 2B). In contrast, the RFP expression level was not affected by addition of the GFP-targeted siRNA expression vector, suggesting that the use of RFP is appropriate for correcting the transduction efficiency.

Figure 2C displays the mean fluorescent intensities (MFIs) for GFP within RFP-positive cells. The solid and dotted lines represent GFP expression with or without the GFP expression vector, respectively, while the shaded line represents GFP expression in RFP-positive cells transduced with the siRNA expression vector. The MFIs for each condition were: 5.06 for pRFP only, 226 for pMIG + pRFP, and 37.5 for siVM2GFP + pMIG + pRFP. The siRNA efficiency was calculated as $85.3 \%$, according to the following equation: siRNA efficiency $=$ (MFIpMIG + pRFP MFIpMIG+pRFP+siVM2) / (MFIpMIG+pRFP - MFIpRFPonly). These results suggest that the GFP expression level within RFP-positive cells could be examined at the single cell level by flow cytometry. 
Using this system, we assessed three different siRNA sequences targeting the mouse CREM protein. CREM is a member of the cyclic AMP responsive element binding (CREB) protein family of transcription factors. The siRNAs were designed to target the mouse CREM (NM_013498) coding sequence at 308-, 469-, and 817-bp. As a non-specific siRNA control, we used siRNA targeted to firefly luciferase (GL3, Promega). Figure 3A shows the GFP and RFP expression levels in cells transduced with a GFP expression vector without mouse CREM sequences (pMIG). None of the siRNAs affected the GFP or RFP expression levels (Fig. 3A, left panel). The solid and dotted lines in Fig. 3A (right panels) represent the GFP expression in RFP-positive cells with or without pMIG, respectively. The shaded lines represent the GFP expression levels with transduction of the siRNA expression vectors. The GFP expression within RFP-positive cells was not repressed by any siRNA vector.

Next, HEK293 cells were transduced with pRFP and a bicistronic CREM expression vector (pMIG-mCREM), with or without an siRNA expression vector. CREM-targeted, but not luciferase-targeted, siRNAs specifically repressed GFP expression (Fig. 3B, left panels). The GFP expression levels of RFP-positive cells are plotted in the right panels of Figure 3B. The solid and dotted lines represent GFP expression within RFP-positive cells transduced with or without pMIG-mCREM, respectively. The shaded lines represent GFP expression with transduction of the siRNA expression vectors. The MFIs for each condition were as follows: 6.19 for pRFP only, 59 for pMIG-mCREM + pRFP, 46.4 for pMIG-mCREM + pRFP + siVM2GL3, 9.59 for pMIG-mCREM + pRFP + siVM2CREM\#1, 8.88 for pMIG-mCREM + pRFP + siVM2CREM\#2, and 14.8 for pMIG-mCREM + pRFP + siVM2CREM\#3 (Fig. 3B, right panels). All three siRNA sequences targeting mouse CREM (CREM\#1, \#2, and \#3) effectively suppressed GFP expression in RFP-positive cells compared to the control (no siRNA expression vector), with efficiencies of 93.5\%, 94.9\%, and 83.6\%, respectively. The siRNA for firefly luciferase did not affect GFP expression. 
These results suggest that the effects of the siRNA vectors on the GFP expression level were specific to the CREM sequence located in front of the IRES sequence, and not to the GFP sequence.

To verify our screening method, we measured the mRNA and protein expression levels using real time PCR and Western blotting, respectively (Fig. 4B-D). The total RNAs and proteins for the measurement were extracted from the same samples as used in the flow cytometric analysis. Both the mRNA and protein expression levels of CREM correlated very well with the relative MFI for GFP in RFP-positive cells (Fig. 4E and F, R2 $=0.9537$ and 0.9171 , respectively). We also verified the feasibility of the system by applying it to four other genes (data not shown). 


\section{Discussion}

In this study, we successfully evaluated the efficacy and specificity of siRNAs by monitoring the fluorescent proteins expressed from bicistronic vectors by flow cytometry.

Various reporter genes have been utilized for siRNA screening (20-24). The advantages of using targeted reporter genes over direct measurements are many. For instance, the siRNA efficiency can be evaluated even when target gene-specific reagents such as antibodies are not available. Furthermore, when a target gene is expressed at very low levels by the cells, the effect of an siRNA can be difficult to evaluate by direct measurement of the RNA or proteins. Enhancement of target gene expression by vectors enables us to avoid such problems. Low transduction efficiencies also complicate the evaluation of the effects of an siRNA. Direct measurements typically assess the gene expression level in a cell mass, and not on an individual cell basis. By using flow cytometry, fluorescent proteins of multiple wavelengths can be monitored simultaneously after the simple manipulation of cells (24). The intensities of the multiple fluorescences from a single cell can be quantitatively monitored and recorded. In the present study, fluorescence intensities representing the efficacy of siRNAs (GFP) and the transduction (RFP) were monitored simultaneously from a single cell by flow cytometry, resulting in single cell-based quantification. By monitoring the GFP level of RFP-positive cells, problems arising from low transduction efficiencies could be avoided. Flow cytometric analyses were performed $24 \mathrm{~h}$ after transfection, and did not require RNA or protein extractions, which are time- and labor-consuming processes. Cells were simply treated with trypsin and EDTA and then directly analyzed. Each process step was very simple and was performed within a fairly short time. These features of the experimental process are suitable for high-throughput assay. We utilized bicistronic expression vectors to indicate target gene expression rather than target-fused fluorescent reporter genes. Bicistronic vectors are already widely used in many fields, and cloning is easier to perform than the fusion process. The stability of GFP expressed from a bicistoronic vector 
is theoretically constant irrespective of the target genes. Optimization of the time course of the experiment is unnecessary because of the consistency of protein stability. In addition, GFP repression by an siRNA against a target gene is mediated at the mRNA level and not after translation, since the target gene and GFP are transcribed as a single mRNA and are translated in different ways (cap-dependent and -independent translation, respectively) $(25,26)$. Therefore, RNA interference-dependent gene silencing can be more precisely monitored than is possible using the fusion system.

In summary, we have devised a novel flow cytometry-based method to evaluate the effects of siRNAs. Cell samples for flow cytometry are prepared by transducing siRNA and a bicistronic vector, which expresses GFP and the target gene, together with an RFP expression vector. The simplicity of this flow cytometry-based method will facilitate the screening of effective siRNA sequences.

\section{Funding}

This work was supported by the Mitsubishi Foundation to H.H.; and Kakenhi [grant numbers 21591246 to H.H. and 21590443 to J.I.].

\section{Acknowledgements}

We thank Drs S Mizuno and K Akashi for generously providing us with siVM2 vector. 


\section{References}

1. Fire, A., Xu, S., Montgomery, M.K., Kostas, S.A., Driver, S.E. and Mello, C.C. (1998) Nature, 391, 806-811.

2. Dykxhoorn, D.M., Novina, C.D. and Sharp, P.A. (2003) Nat Rev Mol Cell Biol, 4, 457-467.

3. Tuschl, T. and Borkhardt, A. (2002) Mol Interv, 2, 158-167.

4. Paddison, P.J., Silva, J.M., Conklin, D.S., Schlabach, M., Li, M., Aruleba, S., Balija, V., O'Shaughnessy, A., Gnoj, L., Scobie, K. et al. (2004) Nature, 428, 427-431.

5. $\quad$ Dorsett, Y. and Tuschl, T. (2004) Nat Rev Drug Discov, 3, 318-329.

6. Hannon, G.J. and Rossi, J.J. (2004) Nature, 431, 371-378.

7. Ashihara, E., Kawata , E. and Maekawa, T. (in press) Current Drug Targets.

8. Scherer, L.J. and Rossi, J.J. (2003) Nat Biotechnol, 21, 1457-1465.

9. Holen, T., Amarzguioui, M., Wiiger, M.T., Babaie, E. and Prydz, H. (2002) Nucleic Acids Res, 30, 1757-1766.

10. Du, Q., Thonberg, H., Wang, J., Wahlestedt, C. and Liang, Z. (2005) Nucleic Acids Res, 33, 1671-1677.

11. Schwarz, D.S., Ding, H., Kennington, L., Moore, J.T., Schelter, J., Burchard, J., Linsley, P.S., Aronin, N., Xu, Z. and Zamore, P.D. (2006) PLoS Genet, 2, e140.

12. Reynolds, A., Leake, D., Boese, Q., Scaringe, S., Marshall, W.S. and Khvorova, A. (2004) Nat Biotechnol, 22, 326-330.

13. Amarzguioui, M. and Prydz, H. (2004) Biochem Biophys Res Commun, 316, 1050-1058.

14. Naito, Y., Yamada, T., Ui-Tei, K., Morishita, S. and Saigo, K. (2004) Nucleic Acids Res, 32, W124-129.

15. Ui-Tei, K., Naito, Y., Takahashi, F., Haraguchi, T., Ohki-Hamazaki, H., Juni, A., Ueda, R. and Saigo, K. (2004) Nucleic Acids Res, 32, 936-948. 
16. Li, W. and Cha, L. (2007) Cell Mol Life Sci, 64, 1785-1792.

17. de Felipe, P. (2002) Curr Gene Ther, 2, 355-378.

18. Ngoi, S.M., Chien, A.C. and Lee, C.G. (2004) Curr Gene Ther, 4, 15-31.

19. Hirai, H., Zhang, P., Dayaram, T., Hetherington, C.J., Mizuno, S., Imanishi, J., Akashi, K. and Tenen, D.G. (2006) Nat Immunol, 7, 732-739.

20. Kumar, R., Conklin, D.S. and Mittal, V. (2003) Genome Res, 13, 2333-2340.

21. Du, Q., Thonberg, H., Zhang, H.Y., Wahlestedt, C. and Liang, Z. (2004) Biochem Biophys Res Commun, 325, 243-249.

22. Smart, N., Scambler, P.J. and Riley, P.R. (2005) Biol Proced Online, 7, 1-7.

23. Hung, C.F., Lu, K.C., Cheng, T.L., Wu, R.H., Huang, L.Y., Teng, C.F. and Chang, W.T. (2006) Biochem Biophys Res Commun, 346, 707-720.

24. Ho, H.Y., Cheng, M.L., Wang, Y.H. and Chiu, D.T. (2006) Cytometry A, 69, 1054-1061.

25. Martinez-Salas, E. (1999) Curr Opin Biotechnol, 10, 458-464.

26. Stoneley, M. and Willis, A.E. (2004) Oncogene, 23, 3200-3207. 


\section{Figure Legends}

Figure 1. Strategy and experimental design. The target gene and GFP are transcribed as a single mRNA from a bicistronic vector and translated independently (left panel). An siRNA against the target gene destroys the entire mRNA, including the target gene and GFP expressed from the bicistronic vector. The GFP expression level should reflect the effects of the siRNA (right panel). RFP is expressed from an independent vector.

Figure 2. Flow cytometry-based quantification of GFP expression using GFP-targeted siRNA. HEK293 cells were transduced with an RFP expression vector, co-transduced with or without a GFP and/or an siRNA expression vector, as indicated. Fluorescence was monitored $24 \mathrm{~h}$ after transduction by fluorescent microscopy (A) or flow cytometry (B and C). The mean fluorescent intensities (MFI) of GFP in the RFP-positive cells were plotted in panel C. The solid and dotted lines represent GFP expression within RFP-positive cells with or without the GFP expression vector, respectively. The GFP expression levels with the siRNA expression vectors are represented by the shaded lines. Results are representative of three independent experiments.

Figure 3. Flow cytometry-based method for screening for an effective siRNA against the mouse CREM gene. (A) HEK293 cells were transduced with an RFP vector and a GFP expression vector with or without one of the siRNA expression vectors (firefly luciferase [GL3], CREM\#1, CREM\#2, or CREM\#3). Flow cytometry data analyzed $24 \mathrm{~h}$ after transduction are shown in the left panels. The RFP-positive cells (left panels) were analyzed for GFP expression (right panels). The solid and dotted lines represent GFP expression within RFP-positive cells with or without pMIG, respectively. (B) HEK293 cells were transduced with the pRFP vector and a bicistronic CREM expression vector (CREM+GFP) with or without one of the siRNA expression vectors (firefly luciferase [GL3], 
CREM\#1, CREM\#2, or CREM\#3). Results are representative of three independent experiments.

Figure 4. Verification of the flow cytometry-based method by conventional methods. HEK293 cells were transduced with an RFP vector and a bicistronic CREM expression vector (CREM+GFP), with or without one of the siRNA expression vectors (firefly luciferase [GL3], CREM\#1, CREM\#2, or CREM\#3). (A) Flow cytometric analysis $24 \mathrm{~h}$ after transduction. Mean fluorescent intensities of GFP in RFP-positive cells. (B) Relative expression level of mRNA for the mouse CREM gene, normalized to GAPDH. (C and D) Western blotting results. The band densities in (D) were quantified using Image $\mathrm{J}$ software $(\mathrm{C})$. $(\mathrm{E}$ and $\mathrm{F})$ The MFI was correlated with the mRNA (R2 = 0.9537) $(\mathrm{E})$ or protein expression $(\mathrm{R} 2=0.9171)(\mathrm{F})$. Results are representative of three independent experiments. 


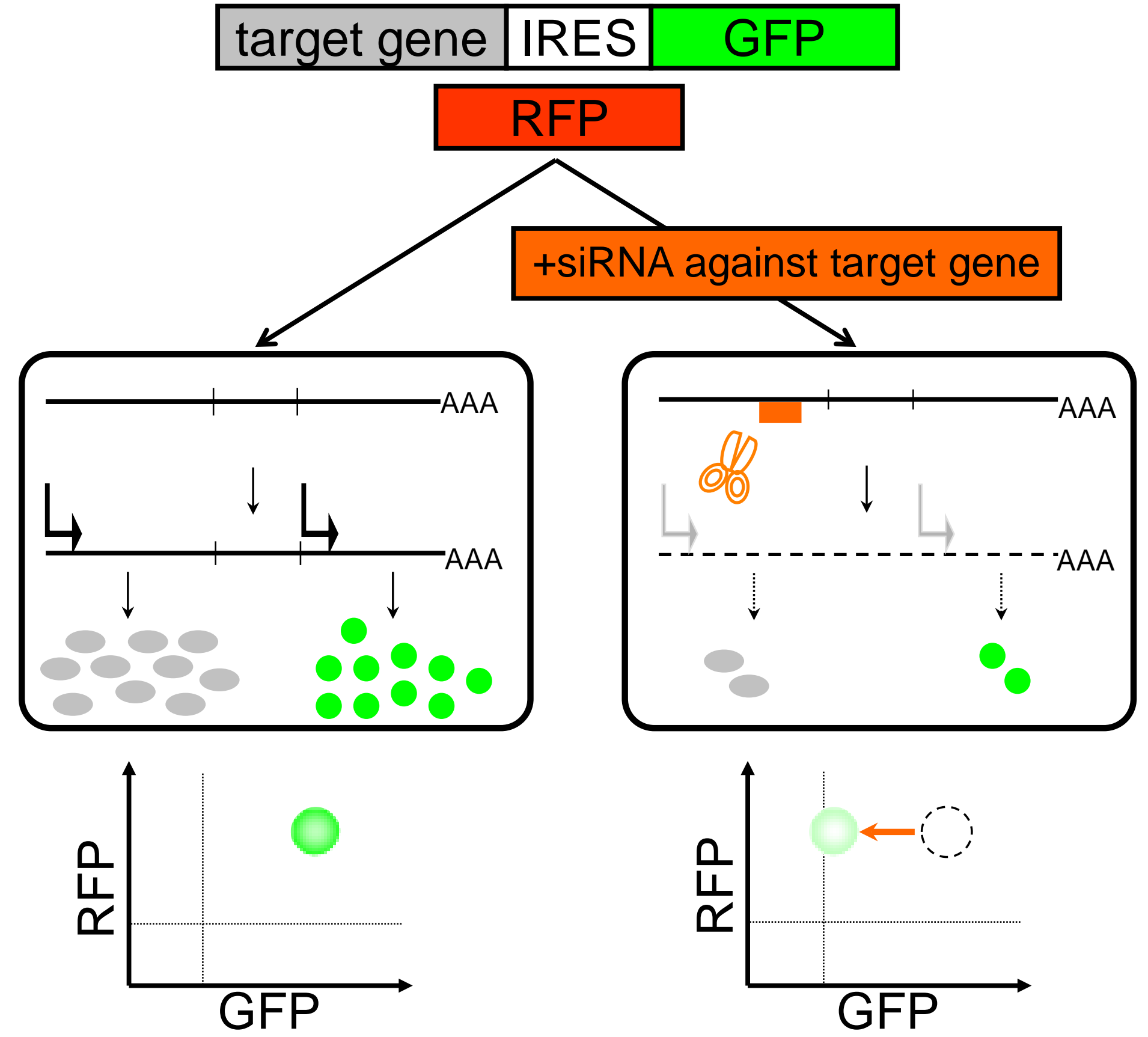


A

B

C

\section{GFP SiRNA}
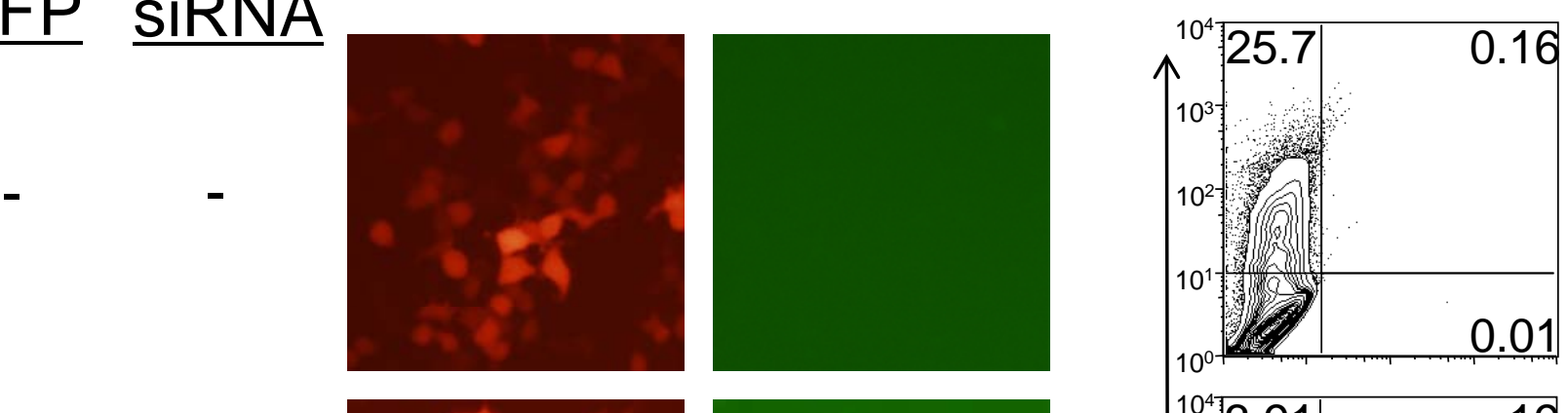

In $\mathrm{RFP}^{+}$cells
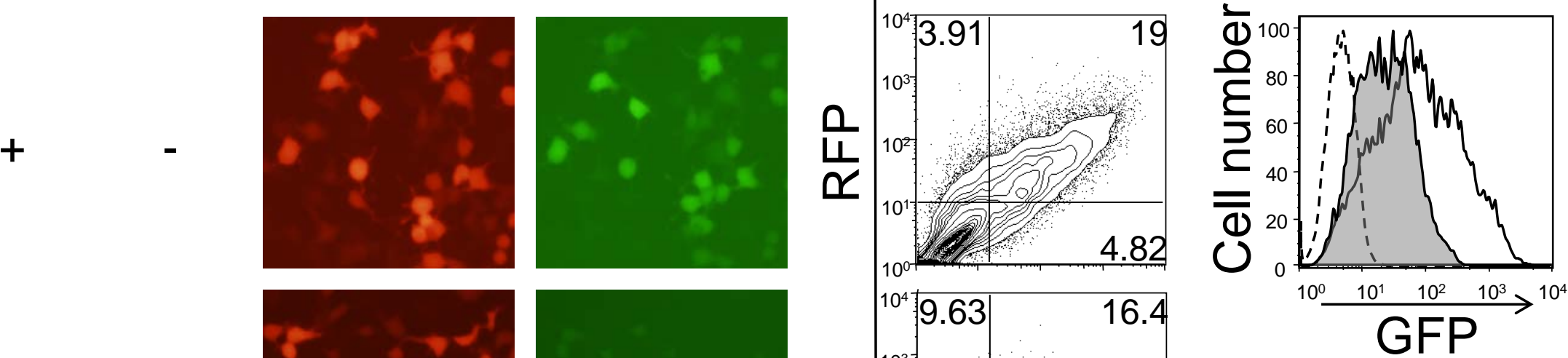

$+$
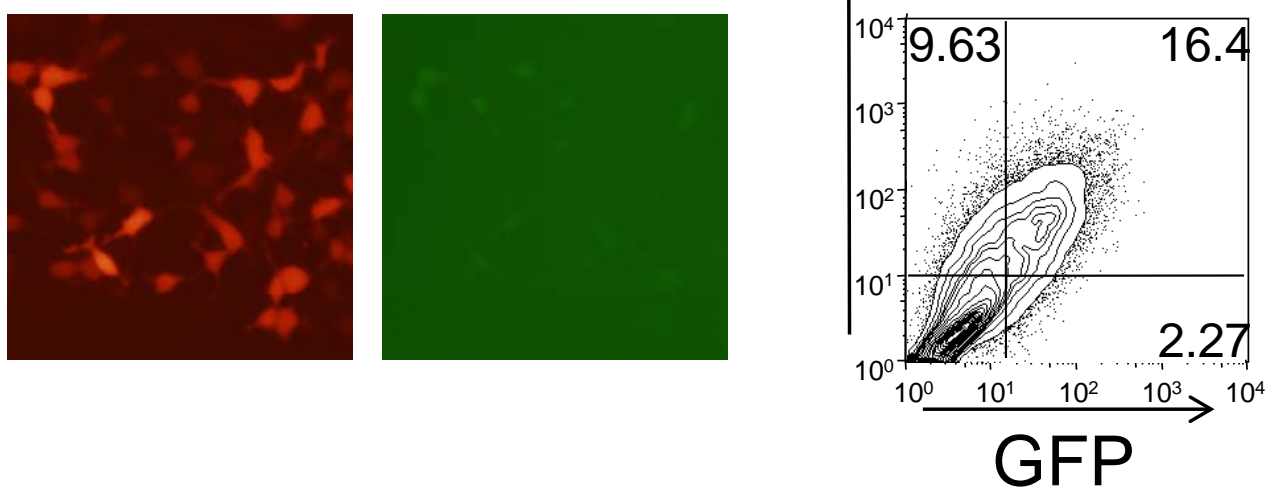
A

B
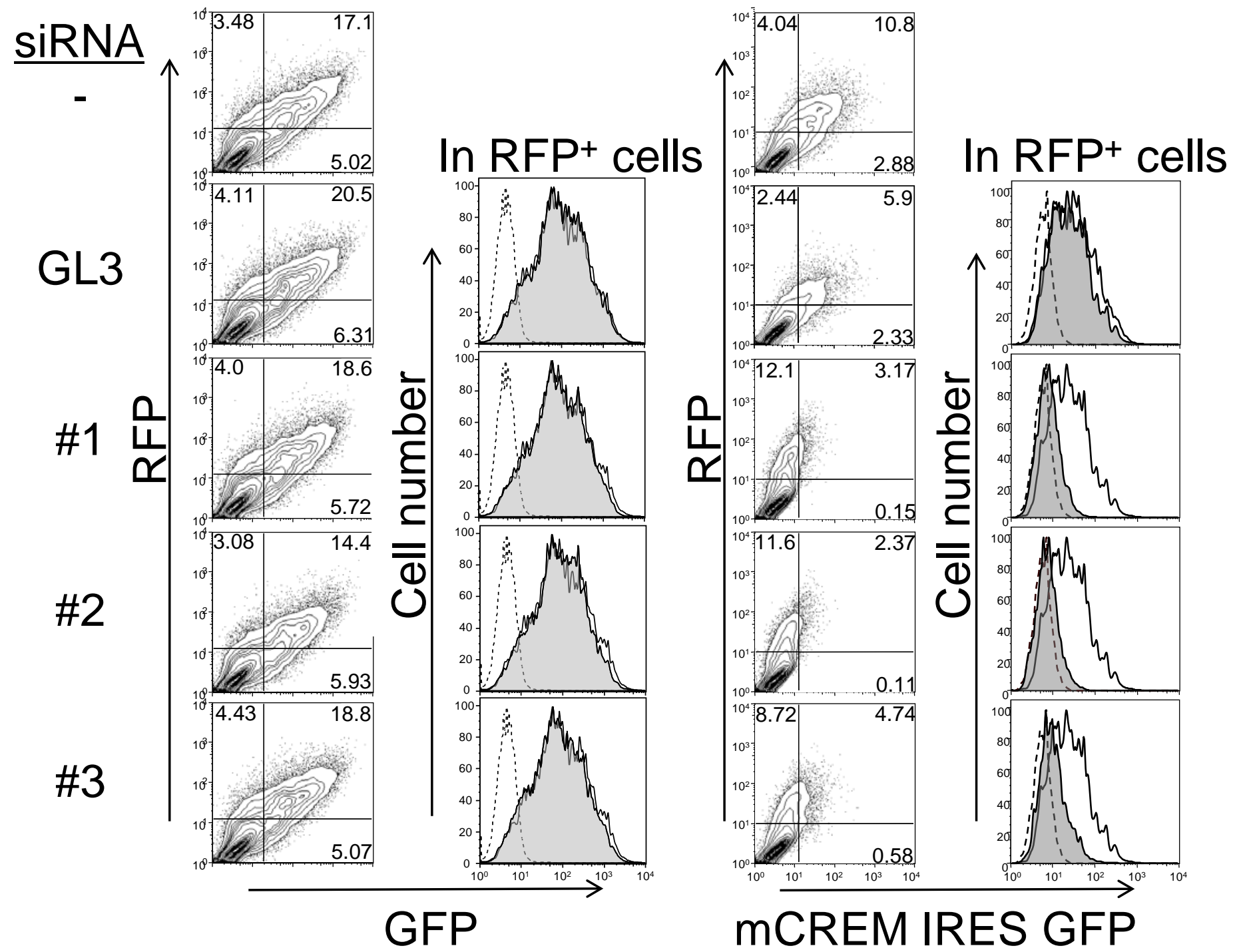

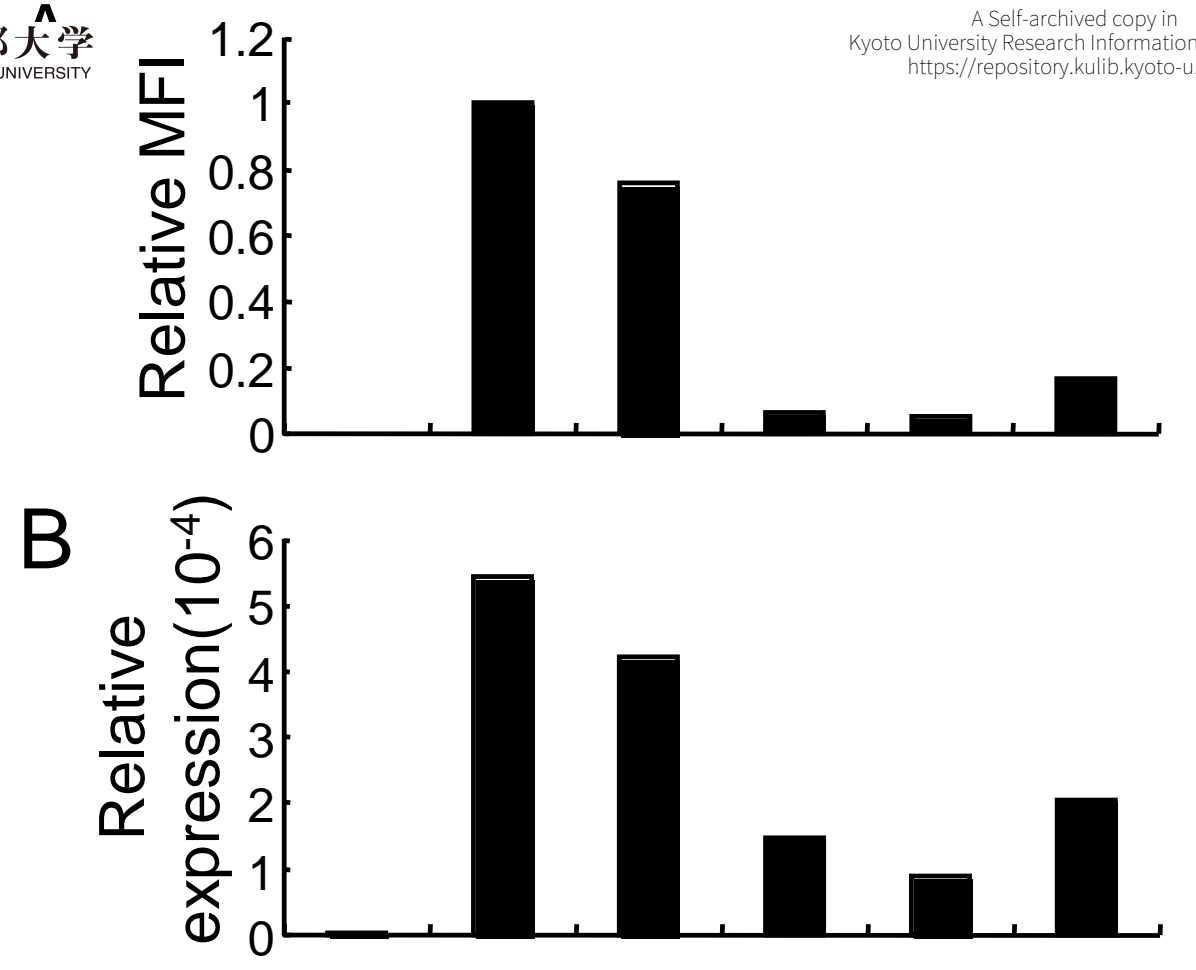

C
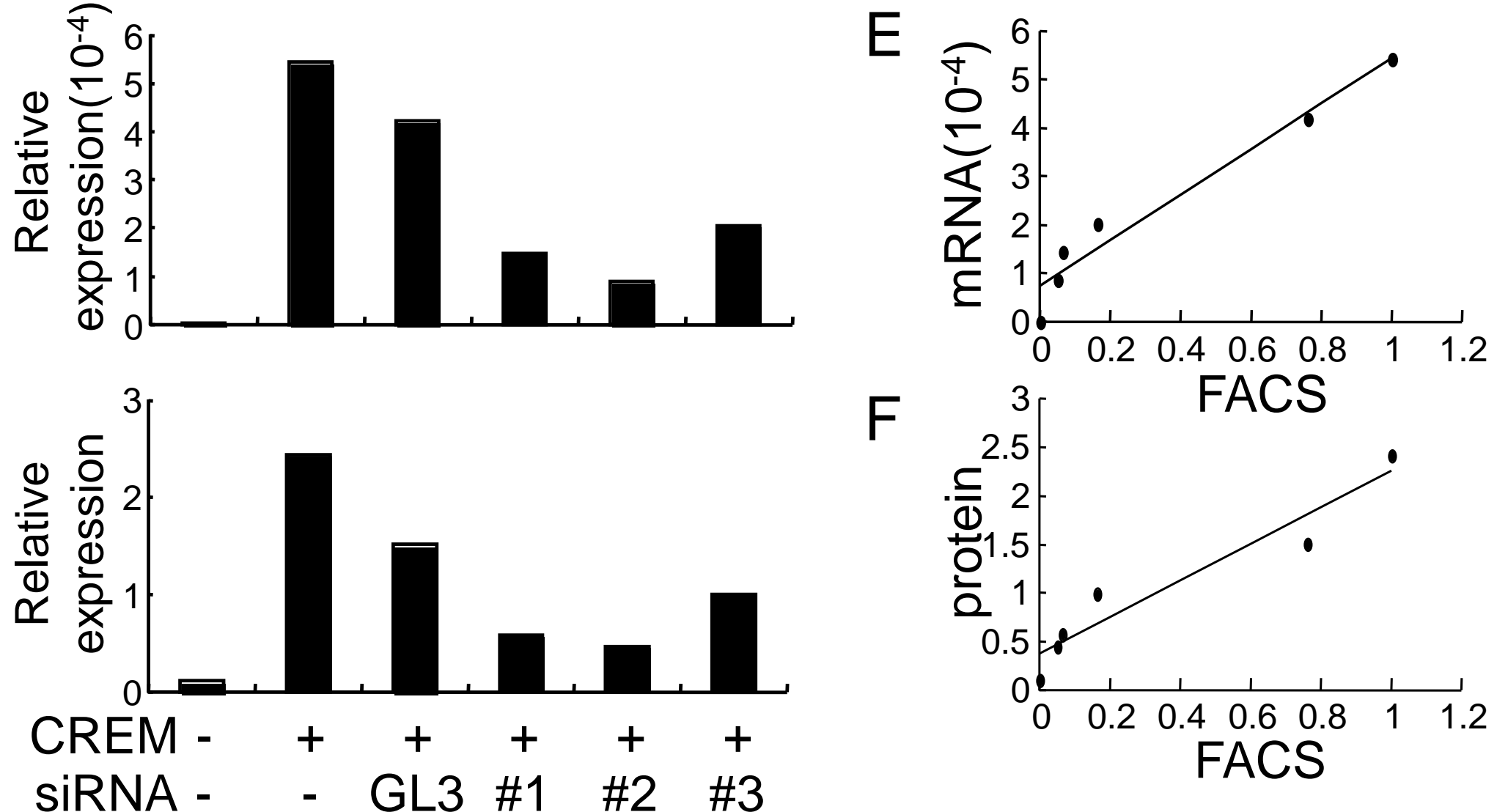

D

$m-\infty-$ CREM 